\title{
Testing a model of adaptation in adolescents and young adults (AYA) with spina bifida (SB)
}

\author{
Kathleen J Sawin ${ }^{1 *}$, Timothy J Brei ${ }^{2}$, Amy Heffelfinger ${ }^{3}$, Susan E Cashin ${ }^{4}$, Thomas S Webb ${ }^{5}$, Constance F Buran ${ }^{6}$ \\ From 54th Annual Meeting of the Society for Research into Hydrocephalus and Spina Bifida \\ Vancouver, Canada. 7-10 July 2010
}

\begin{abstract}
Background
Little is known physical health $(\mathrm{PH})$, mental health $(\mathrm{MH})$ and health-related quality of life (HRQOL) adaptation outcomes in AYA with SB, and the factors associated with them. The purpose of this presentation is to 1 ) describe the PH (functional status [FS], self-management, secondary conditions), $\mathrm{MH}$ (depression, overall selfworth, and internal/external behavior problems [IBP, EBP]), and HRQOL outcomes; and 2) delineate the direct and indirect relationships of risk and protective variables in the Ecological Model of Secondary Conditions and Adaptation in SB associated with these outcomes. This model includes SB Context (SES, shunt status and level of lesion [LOL]), neuropsychological risk [NPR], and protective processes (adolescent resilience and family resourcefulness).
\end{abstract}

\section{Material and methods}

This is an interdisciplinary descriptive cross-sectional study. AYA were between 12-25 years of age and from 4 clinical programs in the USA. They had no other major medical condition, no mental retardation, were English speaking and functioned at approximately grade levels. Parent and AYA report of $\mathrm{PH}$ and $\mathrm{MH}$ and AYA report of HRQOL outcomes were included. Study measures had reliability and validity data and most had been used in our previous studies. Structural Equation Modelling (SEM) analyses were used to assess the model.

\section{Results}

Mean parent and AYA basic functional status scores reflect need for supervision; self-management reflects need for substantial assistance. Mean parental report of IBP are

\footnotetext{
* Correspondence: sawin@uwm.edu

${ }^{1}$ College of Nursing and Children's Hospital of Wisconsin, UW-Milwaukee,

Box 413, Milwaukee, Wi 53201, USA

Full list of author information is available at the end of the article
}

higher than EBP and both are in the normative range. However, 13-18\% of parent or AYA reports of AYA depressive symptoms were in the clinically significant range.

Self-worth and HRQOL were both moderately high. Three SEM analyses revealed different predictive patterns for $\mathrm{PH}, \mathrm{MH}$, and HRQOL constructs. In the $\mathrm{PH}$ model only LOL was significantly related to FS. NPF mediated the impact of shunt status and LOL on selfmanagement, and age, NPR, and functional status had direct relationships to self-management. In the $\mathrm{MH}$ model, SES and family resourcefulness had indirect paths to $\mathrm{MH}$; whereas AYA resilience, secondary conditions, and age had direct paths to MH. In the HRQOL model SES, age, and family resourcefulness had indirect paths to HRQOL through AYA resilience and $\mathrm{MH}$; whereas AYA resilience and $\mathrm{MH}$ had direct paths.

\section{Conclusions}

Different direct and indirect relationships of the conceptual framework variables were identified for the three outcomes. Clinical interventions should be differentiated by outcome and targeted to risk and protective variables.

\section{Author details \\ ${ }^{1}$ College of Nursing and Children's Hospital of Wisconsin, UW-Milwaukee, Box 413, Milwaukee, Wi 53201, USA. ${ }^{2}$ School of Medicine, Developmental Medicine Department, Indiana University, Indianapolis, INd; Riley Hospital for Children, 702 Barnhill Dr. Indianapolis, IN 46202, USA. ${ }^{3}$ Department of Neurology, Division of Neuropsychology, Medical College of Wisconsin, 9200 W. Wisconsin, Ave, Milwaukee, Wi 53226-3596, USA. ${ }^{4}$ College of Health Sciences, University of Wisconsin-Milwaukee, Box 413, Milwaukee, Wi, USA. ${ }^{5}$ Huntersville Pediatrics and Internal Medicine, Presbyterian Novant Medical Group, 17220 Northcross Dr., Huntersville, NC 28036, USA. ${ }^{6}$ Ambulatory Administration, Riley Hospital for Children, 702 Barnhill Dr, ROC 3205 Indianapolis, IN 46202, USA.}

Published: 15 December 2010

doi:10.1186/1743-8454-7-S1-S24

Cite this article as: Sawin et al:: Testing a model of adaptation in adolescents and young adults (AYA) with spina bifida (SB). Cerebrospinal Fluid Research 2010 7(Suppl 1):S24.

\section{Biomed Central}

(c) 2010 Sawin et al; licensee BioMed Central Ltd. This is an open access article distributed under the terms of the Creative Commons Attribution License (http://creativecommons.org/licenses/by/2.0), which permits unrestricted use, distribution, and reproduction in any medium, provided the original work is properly cited. 